\title{
A Review Paper on Different Application of Genetic Algorithm for Mobile Ad-hoc Network (MANET)
}

$\underline{\text { https://doi.org/10.3991/ijoe.v16i05.13325 }}$

\author{
Jinfa Shi, Misbah Habib ${ }^{(凶)}$, Hai Yan \\ North China University of Water Resources and Electric Power, \\ Zhengzhou, China \\ misbah.habib11@yahoo. com
}

\begin{abstract}
A Genetic algorithm is a search algorithm depends on the methodology of natural selection and natural genetics. A Mobile Ad hoc network (MANET) is a type of wireless nodes (Devices) which are free to move anywhere in the network without any constraints. The nodes which are in range can communicate each other through radio waves and those who are not in range use any routing algorithm for communication. In this review paper, we focus on the problems of MANET that has been solved by applying GA for it and highlights the characteristic and challenges of MANET in the literature. More specifically, we present the summary of review papers and basic solutions that use and in the last, we present some future direction. Consequently, we concluded that modification in Fitness function (Evaluation function) according to the problem is the base of Genetic algorithm and variation in algorithm parameters can give solutions in a reasonable time.
\end{abstract}

Keywords - Genetic Algorithm, fitness function, parameters, MANET.

\section{Introduction}

The core behind communication between electronic devices using wireless network without any fixed infrastructure has gained active attention of researchers since its reign. The increase developments of portable devices and tablets made a possible world of remote (wireless) that can share information without any wire connection. Developing a real world of mobile ad-hoc network is not an easy task because of its complications and need high investment in hardware. But there is many simulators (software) like MATLAB, NS-2, NS-3 and OMNET++ that use to evaluate the performance of this network. Numerous executions have been displayed and analyzed to improve the operations of wireless network according to the different situations. MANET is emerging technology in the wireless network world. Mobile Ad-hoc Networks (MANETs) are extraordinary sort of system in which the portability of the nodes is very high. All nodes should be equivalent in handling power. Mobile ad- hoc systems are the system which includes nodes without framework. The system over radio connections are associations almost since of the self-association of the flexible devices (hubs). Explicit guiding conventions are needed for building up courses among the nodes. The nodes in an 
Ad hoc network can perform both actions at a time like as both routers and hosts [1]. Evolutionary algorithms are metaheuristic algorithms intended to solve complex optimization problems [2]. Although they were proposed several decades ago, their application to real optimization problems requires high computational resources that were not available at that time. Nowadays, modern computers and laptops based on multicore architectures are capable of running millions of operations per second, making the application of evolutionary algorithms a reality in many scientific areas. They are based on the evolution of a population of potential solutions by the mean of genetic operators like crossover and mutation [2].GA is an evolutionary optimization approach, they are particularly applicable to problems which are large, non-linear and possibly discrete in nature [4]. Evolutionary algorithms have been successfully applied to many mobile multichip problems such as topology management, routing protocols and mobility models and many others. GA try to work on the principle of natural selection, as in natural selection over the time individuals with "good" genes survive, whereas "bad" ones are rejected [4].GA collects the possible alternative solutions of a problem as a genetic string [4]. A set of chromosomes that forms a population, is ranked by fitness (evaluation) function. The fitness evaluation function plays a critical role in GAs because it provides information how good is each candidate [4]. The initial population is usually generated at random [4]. The evolution from one generation to the next one involves mainly three steps: fitness evaluation, selection and reproduction [5].

In this paper, we will review different papers that used Genetic algorithm. A Second section of the paper includes a literature review about MANET evolution, characteristic and application and evolutionary algorithm (Genetic algorithm). Third section contains review summary, methodologies of different authors used in paper and simulation results. The Fourth section of the paper contains tables that cover full summary, network type of all reviewed paper. Next, we have given some future direction. Last part included conclusions.

\section{Literature Review}

In the past two decades, the progress of wireless technology and the increasing use of small portable computers and devices have made wireless network so popular. Wireless networks allow hosts to roam without the constraints of wired connection and users are free to move independently in any direction, while at the same time being connected to the network. The deployment of wireless network can be difficult in a condition where there is no fixed infrastructure or the local infrastructure is not reliable. In these situations, the mobile Ad hoc network can be considered as a better choice [6], [7]. In the next section of literature, we present about MANET and its evaluation, characterization, applications and challenges. Detail about Evolutionary algorithm (Genetic algorithm). 


\section{MANET}

MANET stands for (Mobile ad hoc Network) also called as wireless ad-hoc network or ad-hoc wireless network that usually has a routable networking environment on top of a Link Layer ad hoc network [8]. Mobile ad-hoc network (MANET) is a wireless network without any fixed infrastructure such as a base station. These nodes generally have a restricted transmission range and so, each node search for the assistance of its neighboring nodes in forwarding packets. The main challenge for the MANET is to equip each device to continuously maintain the information required to properly route traffic [8]. In Mobile ad-hoc networks (MANETs) present complicate distributed systems that depends on wireless mobile nodes that can freely and dynamically self-organize into arbitrary and temporary ad hoc network topologies, allowing people and devices to absolute internetwork in areas with no precedent communication infrastructure. Broadcasting in MANET presents more challenges than in wired networks as a consequence of node mobility and less system resources. MANETs can be applied in any situation where the deployment of a fixed communication infrastructure is not possible. A clear example of this scenario is disaster scenario, where the fixed communication infrastructure is likely to be destroyed or malfunctioning. [9]. Each node is equipped with a wireless transceiver. Each node may communicate directly with each other even in out of range conditions with intermediate nodes performing the routing functions. Thus, a node doesn't only play the role of a data terminal, but also acts as a router, which sends packets to desired nodes. The Mobile Ad hoc network has many applications, the most common mentioned are crisis management and military operations; in additions to its application in collaborative and distributed computing, wireless mesh control, wireless sensor network, hybrid control and many more [10].

\subsection{Evolution of MANET}

- In 1970, Norman Abramson and his fellow researchers at the University of Hawaii invented ALOHA net.

- In 1972, early ad-hoc networking applications can be traced back to DARPA Packet Radio Network (PR Net) project, which was primarily inspired by the efficiency of the packet switching technology.

- In 1980, Survivable Radio Networks (SURAN) was developed by DARPA to address the main issues in PR-Net, in the areas of network scalability, security, processing capability, and energy management.

- During 1983, with the emergence of Internet Engineering Task Force (IETF) formed the mobile ad hoc networking group.

- In 1994, to leverage the global information infrastructure in the mobile wireless environment. Department of Defense (DOD) initiated the DARPA Global Mobile (GloMo) [11]. 


\subsection{MANET characteristics}

- Distributed operation: There is no background network for the central control of the network operations, the control of the network is distributed among the nodes. The nodes involved in a MANET should cooperate with each other and communicate among themselves and each node acts as a relay as needed, to implement specific functions such as routing and security.

- Multi hop routing: When a node tries to send information to other nodes, which is out of its communication range, the packet should be forwarded via one or more intermediate nodes.

- Autonomous terminal: In a MANET, each mobile node is an independent node, which could function as both a host and a router.

- Dynamic topology: Nodes are free to move arbitrarily with different speeds; thus, the network topology may change randomly and at unpredictable times. The nodes in the MANET dynamically establish routing among themselves as they travel around, establishing their own network.

- Light-weight terminals: In maximum cases, the nodes at MANET are mobile with less CPU capability, low power storage and small memory size.

- Shared Physical Medium: The wireless communication medium is accessible to any entity with the appropriate equipment and adequate resources. Accordingly, access to the channel cannot be restricted [12].

\subsection{MANETs challenges}

- Limited bandwidth: Wireless link continues to have significantly lower capacity than infrastructure networks. In addition, the realized throughput of wireless communication after accounting for the effect of multiple access, fading, noise, and interference conditions, etc., is often much less than a radio's maximum transmission rate.

- Dynamic topology: Dynamic topology membership may disturb the trust relationship between nodes. The trust may also be disturbed if some nodes are detected as compromised.

- Routing Overhead: In wireless ad hoc networks, nodes often change their location within the network. So, some stale routes are generated in the routing table which leads to unnecessary routing overhead.

- Hidden terminal problem: The hidden terminal problem refers to the collision of packets at a receiving node due to the simultaneous transmission of those nodes that are not within the direct transmission range of the sender, but is within the transmission range of the receiver.

- Packet losses due to transmission errors: Ad hoc wireless networks experience a much higher packet loss due to factors such as increased collisions due to the presence of hidden terminals, presence of interference, uni-directional links, and frequent path breaks due to mobility of nodes. 
- Mobility-induced route changes: The network topology in an ad hoc wireless network is highly dynamic due to the movement of nodes; hence an on-going session suffers frequent path breaks. This situation often leads to frequent route changes.

- Battery constraints: Devices used in these networks have restrictions on the power source in order to maintain portability, size and weight of the device.

- Security threats: The wireless mobile ad hoc nature of MANETs brings new security challenges to the network design. As the wireless medium is vulnerable to eavesdropping and ad hoc network functionality is established through node cooperation, mobile ad hoc networks are intrinsically exposed to numerous security attacks [12].

\subsection{MANET applications}

Some of the typical applications include:

- Military battlefield: Ad-Hoc networking would allow the military to take advantage of commonplace network technology to maintain an information network between the soldiers, vehicles, and military information head quarter.

- Collaborative work: In some business environments, the need for collaborative computing might be more important outside office environments than inside and where people do need to have outside meetings to cooperate and exchange information on a given project.

- Local level: Ad-Hoc networks can autonomously link an instant and temporary multimedia network using notebook computers to spread and share information among participants at an e.g. Conference or classroom. Another appropriate local level application might be in home networks where devices can communicate directly to exchange information.

- Personal area network and Bluetooth: A personal area network is a short range, localized network where nodes are usually associated with a given person. Shortrange MANET such as Bluetooth can simplify the intercommunication between various mobile devices such as a laptop, and a mobile phone.

- Commercial Sector: Ad hoc can be used in emergency/rescue operations for disaster relief efforts, e.g. In fire, flood, or earthquake. Emergency rescue operations must take place where non-existing or damaged communications infrastructure and rapid deployment of a communication network is needed [12].

\section{Evolutionary Algorithms for Solving Problems}

EA are stochastic search derived from the classic evolution theory that are implemented on computers in the variety of cases. The basic idea is that if only those individuals of a population reproduce, which meet a certain selection criterion, and the other individuals of the population die, the population will converge to those individuals that best meet the selection criteria. If imperfect reproduction is added the population can begin to explore the search space and will move to individuals that have an increased selection probability and that inherit this property to their descendants. These 
population dynamics follow the basic rule of the Darwinist evolution theory, which can be described in short as the "survival of the fittest" [13].

Types of EA are:

- Genetic programming (GP)

- Evolution strategies (ESs)

- Evolutionary programming (EP).

\section{Genetic Algorithm}

The basic principles of GA's were first laid down rigorously by Holland in 1975 [14]. Using heuristic methods, genetic algorithms (GAs) can find a very good solution, not the best one, to such problems in a reasonable time [15]. They, however, do not guarantee the optimal solution. By borrowing a technique from nature [15]. Genetic algorithm starts with a set of solution called population and one individual solution, called chromosomes. The Solution is taken from a parent population and generate new more useful offspring. Often, the definition of a criterion of goodness/fitness of a solution is also part of the problem and it acts as a uniform measure for evaluating the quality of a solution. To evaluate the fitness of solutions, one must develop a fitness function (criterion) for each solution [15]. This process is inspired by the hope that the current offspring will be the improvement of the old parent. New offspring is treated as new parent who are chosen to design new population, which are selected according to their fitness value [14]. In every step of Genetic algorithm, the chromosomes will undergo many genetic operators like crossover, selection and mutation [14]. The outcome of genetic operation will become the solution of subsequent generations. These steps go on until any solution is achieved or terminating state is found. The main idea behind GA is to find the highly optimizes solution. At the same time GA maintains varieties, so that it can find from huge search space [16]. Those individuals which are most successful in surviving and attracting mates will have a relatively large number of offspring's. Poorly performing individuals will produce little or even no offspring at all [17].

\subsection{Applications of GA for solving MANET problems}

A genetic algorithm (GA) is a meta heuristic computational method [19], inspired from biological evolution [20], that aims to imitate the robust procedures used by various biological organisms to adapt as part of their natural evolution. GAs have been successfully used in fields as diverse as aircraft industry, chip design, computer animation, drug design, telecommunications, software creation, and financial markets [18]. They work with a population of individuals, each representing a possible solution to a given problem [18]. Genetic algorithm uses its principles of genetics. Takes each problem in a shape of chromosomes. It varies with the problem whether the chromosomes have been taken in bit strings, float, and double, permutation and character. Fitness function has an important role in algorithm, which has been formulated to keep the 
variety of problems in mind. These things make this algorithm more efficient and have been successfully applied to a variety of problems of the MANET.

Let's discuss different research paper, which have applied Genetic algorithm on MANET successfully.

(David Montana) MANET is different according its nature. Author suggested in this paper 'Optimizing Parameters of a Mobile Ad Hoc Network Protocol with a Genetic Algorithm' that MANET are created for uncertain situations where there is difficult to apply a complete wire connection. However, MANAET networking protocols are often designed with many modifiable parameters. In this paper, proposed protocol sends out alert message to the neighbor node to check, its existence in the network. If the neighbor node doesn't reply, that means link is broken. Detecting the other node availability in the network. This task has been accomplished by using LSU (link state update) packet, which transferred all over the network. In this way, each node had already information about other nodes without disturbing the rest of the network. Different parameters have set that works with Genetic algorithm to configure the network.

- Further explanations about protocol "Proactive link state protocol" is the family of network protocol. Author provides the list of GA parameters that allowed to adjust in order to configure the networking protocol that are Heartbeat Interval, Heartbeat Points, Score History Size, Up Score Threshold, Down Score Threshold, Routing Algorithm, Routing Event Interrupt Period, Routing, Global Interrupt Period, Traffic Max Attempts.

Author investigated the performance of Genetic algorithm for automated selection of parameters in MANET.Author uses "weighted sum multiple objective" criteria in the algorithm. Current work in paper focuses on two major points that are: dropped packets and transmission delay.
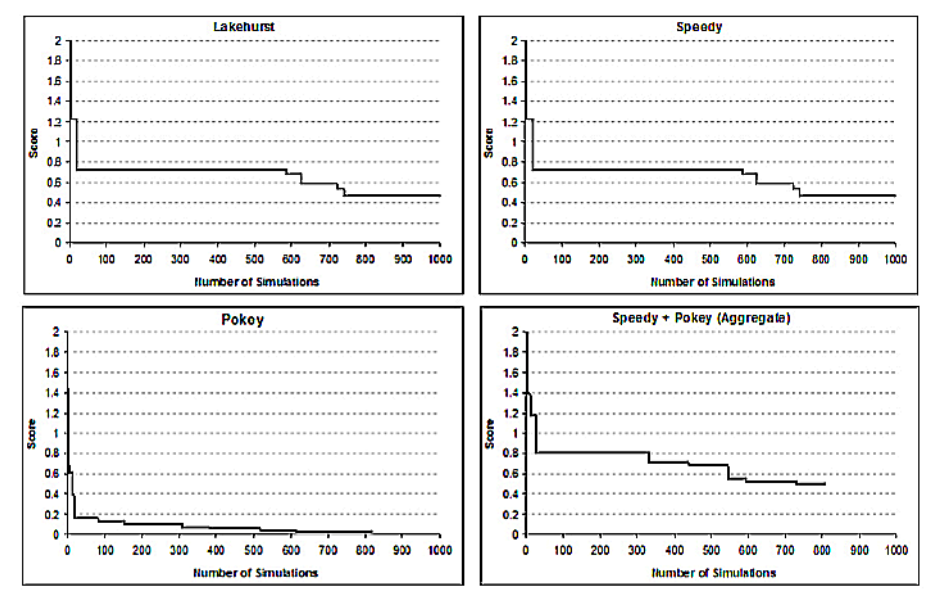

Fig. 1. Simulation results progress of automated optimization on four different datasets 
Simulation results show the performance of the algorithm, which applied to different data sets (Lakehurst, speedy, Pokey and combining speedy and pokey). Results show that Genetic algorithm can optimize different types of operating conditions. Author concluded that automated parameters give better parameter values than others.

(Dr. Ketan Kotech2007) Author suggested that GA is considered the most wellknown technique for multi-objective optimization problems. Routing in MANET is highly expensive due to limited resources, dynamic topology and the variation in the links that are created when nodes (devices) joined the network and disconnected when it left.

- Applications of MANET in different fields of life that are, Day to day information sharing, Disaster relief and Military operations.

- Some challenges for MANET (Mobile ad hoc network) which are:

- All nodes can move in the network without any restriction that caused vulnerable failure.

- And this failure affects the battery drainage or node movement that cannot participate in the network because of topology change.

- Author developed a multi objective function that deal with the QoS (Quality of service) parameters, developed GA (Genetic Algorithm) for main purposes:

- Optimize route that satisfy the QoS parameters.

- Provide an alternative route in case of delay to deal with dynamic topology.

The Basic purpose of this paper to find the best path between nodes with best Quality of service. Author concluded that when other nodes joined this network, its QOS gets slower. This situation has been dealt by genetic algorithm.

(E. Baburaj”,et al, 2008) Author determined in this paper 'An Intelligent Multicast Ad-hoc on demand Distance Vector Protocol for MANET that fastest growing field of the multicast routing protocol in "MANET". As per the nature, MANET is suitable in an unpredictable situation. MANET is complicated due to its dynamic topology. For this problem Genetic Algorithm has been developed by using a spanning tree. Initially random spanning tree with undirected graph has been taken. After applying crossover and mutation of it. A new optimized route has been found, subsequently Author used MAODV protocol that is Ad hoc on demand tree-based protocol. The basic purpose of this protocol to find routes in the network when a node wants to send data. Author present a GA-based MAODV model to support the multicast routing optimization algorithm in mobile ad-hoc networks.

- Author explained the nature of MANET, that protocol, which designed for a traditional network, cannot be used in Mobile ad hoc network because:

- Nodes in ad-hoc networks change frequently.

- Fixed infrastructure does not exist for route computation.

- Restriction in power, converge and bandwidth.

- Further author points out that Topology changing in the network operation causes the problem that are Dynamic topologies, Group membership Interoperability, Mobility, Network resources, Power consumption, Quality of Services (QoS), Reliability and Security, Routing Information and Scalability. 


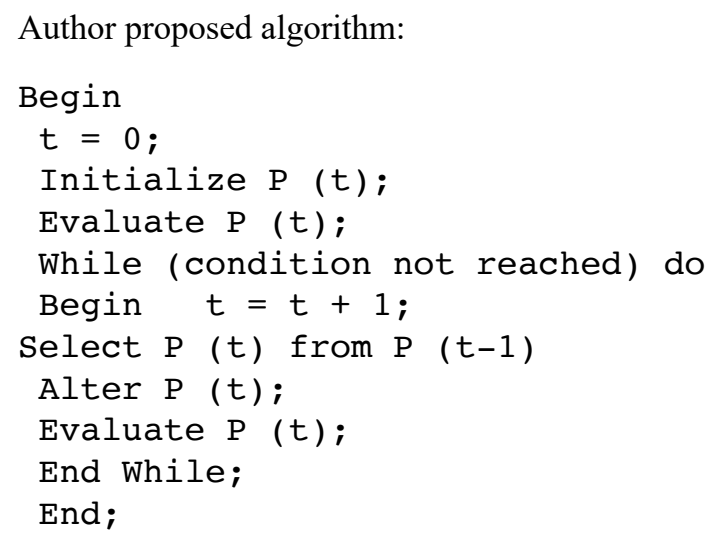

(Suresh Kumar 2011) Author in this paper has presented an efficient method to secure node while in sending and receiving data. When a node wants to send data to destination, first it requests to neighbor node to provide route guides, if neighbor node already has an earlier path as a form of back up, it has been used. Otherwise its responsibility of that node to provide its neighbor node possible path.

Genetic algorithm parameters working in route discovery:

- Initialization

- Fitness function developed by the author is:

$$
f\left(C h_{i}\right)=\left[\sum_{l \in P(s, r)} C_{l}+C_{d}\right]
$$

The Ch. Represents the chromosome fitness value and $\mathrm{Cd}$ the delay time taken by each chromosome where $\mathrm{Cl}$ represents the cost of the path. The above fitness function is being maximized and involves only shortest path and delay constraint, the buffer size for every path is being checked in the evolutionary process.

- Selection scheme, crossover and mutation.

Now its major task to check, the provided path is secure or not. It has been done by using Evolutionary Algorithm (Genetic algorithm). Initial basics, the path which provided on request is taken as a chromosome, after that objective function (Fitness function) has developed that can handle this situation. The purpose of Genetic algorithm to provide an optimal path in case of primary path failure. In order to avoid malicious node, the neighbor node checks the behavior of the next node in the MANET network. To overcome this problem, the real (actual) node has given a special token during the transferring of data between node's source to destination. By using this approach, it can easily recognize if it is real node or not. Further author discovers backup process in case of main (primary) link failure, which are Path discovery phase and Path maintenance.

Author developed self-organized security method that will check the behavior of nodes while transferring data. In this way they have a valid token address in the network. The Process will work as follows:

- Collaborative monitoring 
- Token renewal

- Monitor Routing Behavior

- Monitor Packet Forwarding Behavior

Table 1. Simulation Parameters

\begin{tabular}{|l|l|l|}
\hline \multicolumn{1}{|c|}{ Parameters } & \multicolumn{1}{c|}{ Values } & \multicolumn{1}{c|}{ Descriptions } \\
\hline Simulator & NS2 & Simulator Tool \\
\hline Simulation time & $300 \mathrm{~s}$ & Maximum execution time \\
\hline Simulation area & $500 \mathrm{mx} 500 \mathrm{~m}$ & Physical boundary of the Network \\
\hline Number of nodes & 20 & Nodes participating in the nodes \\
\hline Transmission range & $100 \mathrm{~m}$ & Frequency of the Node \\
\hline Max speed & $0,5,10,15,20 \mathrm{~m} / \mathrm{s}$ & Speed of nodes \\
\hline CBR flows & 20 & Constant Bit Rate link used \\
\hline Data payload & 512 Bytes & Packet size \\
\hline Sending rate & 4 packet/s & Max no of sending packets \\
\hline Movement model & Random waypoint & Network connection \\
\hline
\end{tabular}

(Shenbagarai R2012) Author in this paper explains about MANET Battery life that is limited. Author suggested that selection of nodes should be very careful because all its effect on the life of the multicast tree. Author used prisms algorithm, after that he got two multicast trees. On that tree, applied Genetic algorithm. These trees are used as initial population of the Algorithm. Multicast tree has taken as a chromosome. A file that has been used to simulate nodes are, Tcl files, scenario file and CBR file.

Information got from Trace file that are:

- Contains energy

- Mobility

- Packet transmission details

- Energy and mobility information in two different instances.

Table 2. Parameters and Description for fitness value calculation

\begin{tabular}{|l|l|}
\hline \multicolumn{1}{|c|}{ Parameters } & \multicolumn{1}{c|}{ Description } \\
\hline Btv $(\mathrm{t}):$ & Battery energy percentage in node $\mathrm{v}$ at time $\mathrm{t}$ \\
\hline Res_Btv $(\mathrm{t})$ & Residual battery energy capacity of node $\mathrm{v}$ at time $\mathrm{t}$ \\
\hline Full_Btv & Full battery capacity of node $\mathrm{v}$ \\
\hline$\zeta \mathrm{v}$ & Transmission power of node $\mathrm{v}$ \\
\hline$\Gamma$ & Positive weighting factor \\
\hline
\end{tabular}

The proposed method is applicable to reducing the node degree by replacing nodes with lower energy with nodes with higher energy.

(Arun Birdar, at al, 2014) Here in this paper Author have developed two techniques by using a Genetic algorithm based intelligent routing protocol (Unipath and Multipath) for mobile ad-hoc. Mobile ad hoc is used where it is possible to set up wire's connection (e.g.) Military operation, Natural disaster and remote areas. Genetic algorithm to deal with it very efficiently within limited resources. GA uses GAODV ON demand vector protocol to find the best possible path in case of link failure and also improve the 
performance of the network. The Second technique author suggested that AOMDV to calculate multiple paths during route discovery. The working of the algorithm is that to find the next path in case of first path failure. Its duty to find secondary path.

Author found major requirements of designing a reliable routing protocol in MANETs that are as follows:

- Minimum route acquisition

- Route reconfiguration in very short time

- Loop-free routing

- Distributed routing approach

- Minimum control overhead

- Scalability

- QoS provisioning.

Classification of Routing protocol is:

- Flat Routing protocol:

- Flat routing is further divided into categories:

- Table Driven Routing (Proactive)

- On-demand (Reactive)

Route Table Entries followed in the algorithm are as follows:

- Destination IP Address

- Destination Sequence Number

- Valid Destination Sequence Number flag

- Other state and routing flags (e.g., valid, invalid)

- Network Interface

- Hop Count

- Next Hop

- List of Precursors

- Lifetime (expiration or deletion time of the route)

- Maximum Remaining Energy Capacity During route discovery from the source to the destination.

(Neha Agarwal 2015) Author suggested an efficient technique in this paper to provide energy efficient routing for MANET. By using Genetic algorithm alternative path has also been selected in case of first path failure. Source and destination have been set. Author found some problems with the Mobile ad hoc network:

- Limited wireless transmission range

- Broadcast nature of the wireless medium

- Hidden terminal and exposed terminal problems

- Packet losses due to transmission errors and mobility

- Stimulated change of route

- Battery constraints and security problem 
Author proposed algorithm that overcomes two problems:

- Finding an efficient route to transfer data between nodes of the MANET that consumes less power.

- To provide alternate paths when one route gets failed because of the mobility problem of mobile ad hoc networks.

Working of Genetic Algorithm: Genetic algorithm starts its working and it has been applied on 50 random populations. The Fitness function calculates the cost of each node, which check how much time it will take to transfer data from source to destination. After that algorithms other operator completes their task. When new population is created, their cost is also calculated. This process continues until reaching its required condition that has been set. This algorithm also provides a path that will take less energy for transferring data between nodes. Because of the nature of MANET, Alternative path is very important to reconnect with the network again without disturbing other nodes.

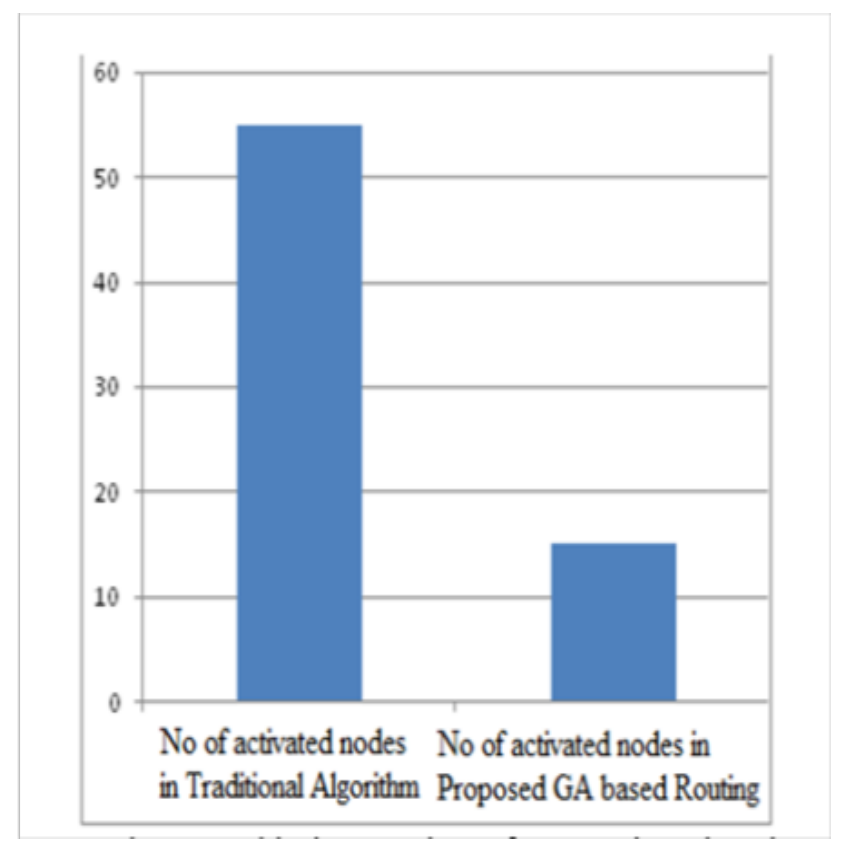

Fig. 2. Graphical result of proposed algorithm

Benefits of using a Genetic algorithm-based protocol:

- Genetic algorithm-based protocol finds many routes from source to destination for sending data.

- So multiple route property is "yes" in this case.

- It makes the system more efficient in case, if there is problem in one route for sending data, alternative route has been provided. 
- Evolutionary techniques of GA make it more versatile to handle the situation in case when more nodes join the network.

(C RAJAN 2015) this paper presents a combination of two algorithms Hybrid Optimization Algorithms (Genetic and Particle swarm optimization) for Multicasting Routing in MANET.A MANET network has been taken as a graph where one variable represents (Set of nodes) and the other represents (Set of links). Working of these two algorithms as, initially population selected by using Roulette wheel selection. Fitness function has calculated which genes have a higher survival chance. These have been selected. Now these solutions have taken as an input of PSO, they compute the velocity of new solution by using sigmoid function. After this PSO reward-based selection is used. Subsequently, all Genetic algorithm operators have completed their work.

A Methodology developed in the paper is:

- This research focuses on a hybrid method combining two heuristic optimization techniques GA and PSO for solving the multicast tree problem. Since GA is encoded using binary values, Binary PSO is used. In Binary PSO, the sigmoidal transfer function is used to filp the binary value.

The pseudo-algorithm of GA in paper is:

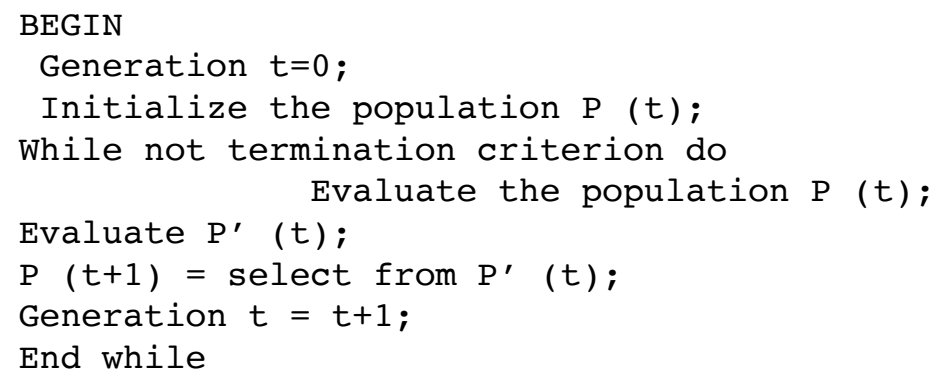




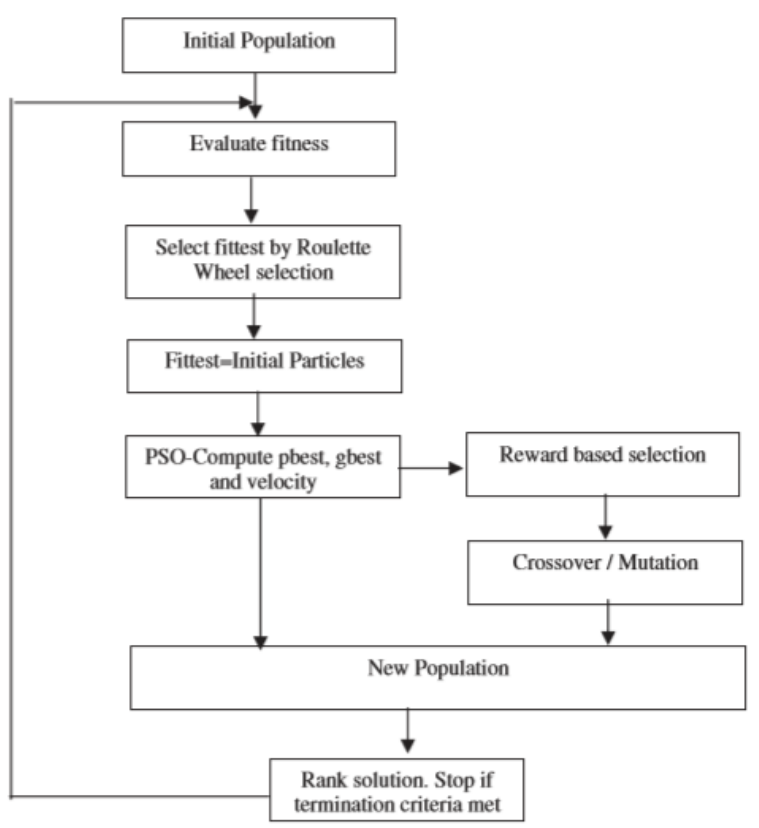

Fig. 3. Flow chart of hybrid algorithm

(Veena Trivedi 2019) Author suggested in her paper "Modified AODV using Genetic algorithm to minimize energy consumption in MANET" that routing in MANET is one of the most challenging and difficult tasks till now. MANET itself is a challengeable due to its dynamic topology and without any centralized network. Many researches provide a different solution to providing data from source to destination. Author suggested that an ad hoc on demand routing protocol is most useable protocol.

Author describes some features of MANET that are:

- Decentralized management

- Resource availability

- Network size

- Compliance amidst nodes

- Dynamic topology

- Confined power supply

The Author's network assumption is as a fellow:

- The network consists of mobile nodes and each node is characterized by a unique Identification number.

- The MANET environment is homogeneous type with all mobile nodes having equal initial energy.

- The number of mobile nodes is application dependent and is assumed as N. 
- The nodes are considered to have mobility characteristics and hence distance between the nodes keeps on varying.

- The distance and the residual energy is considered for calculating a new path between the source and destination.

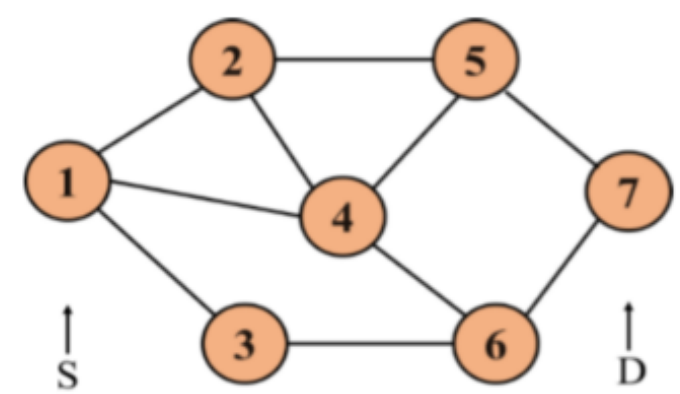

Fig. 4. Distance between source and destination

Some Terminologies Author used in paper are:

- A path from source to destination represents individual population

- An adjacent node corresponding to a node represents as child

- An entire path from source to destination indicates a chromosome

- A string of adjacent nodes identifies a gene

- All the possible paths from source to the destination implies chromosome structure.

Procedure for GA based AODV:

- Step 1: Initial Population: Consider N number of nodes in a network

- Step 2: Initialize all the nodes Step

The neighbor node is selected based on Fitness function.

The Fitness function can be calculated as follows:

$$
\mathrm{Ft}=\mathrm{Fr}+\mathrm{Fd}
$$

Where,

$\mathrm{F}_{\mathrm{t}}=$ Fitness function of each node (depends on residual energy and distance

$\mathrm{F}_{\mathrm{r}}=$ Fitness function based on energy

$\mathrm{F}_{\mathrm{d}}=$ Fitness function of a node based on distance

And is calculated using the formula given in equation 2 and equation 3 .

$$
F_{r}=\frac{E g}{E t}
$$

$\mathrm{E}_{\mathrm{e}}=$ Residual Energy at each node

$\mathrm{E}_{\mathrm{t}}=$ Residual Energy of all the nodes 


$$
F_{d}=\frac{D_{n, n}}{D_{r}}
$$

$\mathrm{D}_{\mathrm{n}, \mathrm{n}}=$ Distance between node to node

$\mathrm{D}_{\mathrm{t}}=$ Total distance from source to Destination

- Step 4: Apply Crossover and Mutation operators are applied in-order to generate the set of paths to the destination

- Step 5: GA is applied with fitness function till a complete path is found. Its stops when all the nodes run out of battery.

Author proposed protocol that uses the Genetic algorithm to resume a path that consumes minimum energy as well as preserve a shortest routing path from source to destination. Performance parameters that proof the efficiency of the protocol.

(Adil 2019) Author suggested a technique to send and receive data that will take a short time and low cost. This paper shows a system that can send and receive data between nodes with minimum loss of data using an evolutionary algorithm called GENETIC ALGORITHM. Author shows the results that can overcome two most factors in any system (cost and time). Author constructed Genetic algorithm to improve the performance of the system.

The authors proposed algorithm working: The GA is searching among a population of points, and it differs from other search methods. Also, it uses data of the objective function without gradient data. The traditional ways use gradient data; wherever the transition of the GA is probabilistic.

- Fitness Function developed by author in the paper is:

$$
f(x)=\frac{1}{1+f(x)}
$$

Basic operators of algorithm used in paper are:

- Elitism

- Selection

- Roulette Wheel Selection

- The crossover

- The mutation 


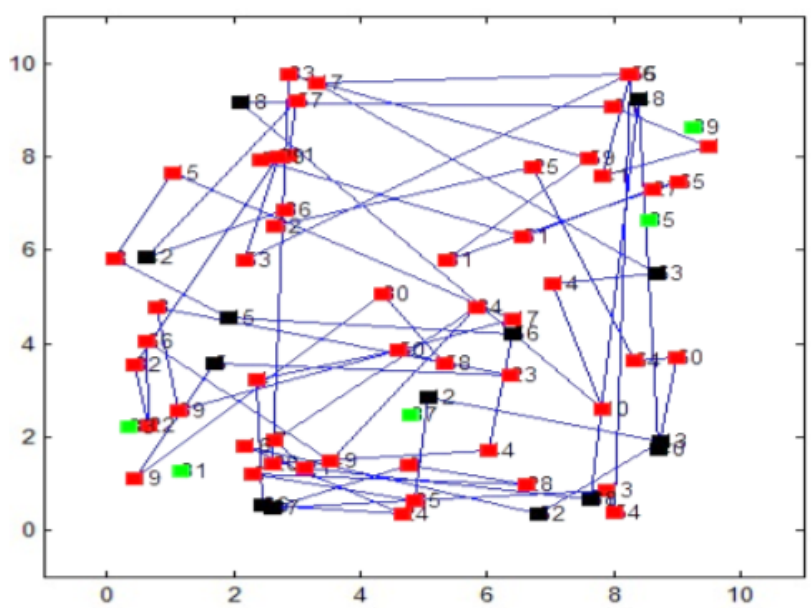

Fig. 5. Generation 200 best path length 235.0194 with number of barge 66

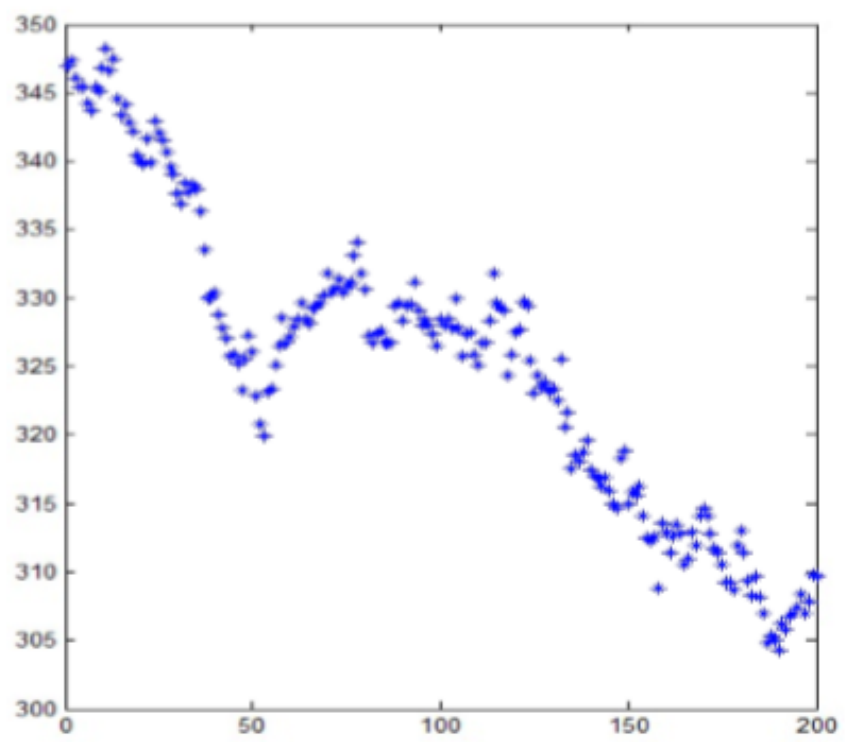

Fig. 6. Show the cost evaluation with number of barge 66

Cost and time problem have been solved by using an efficient Genetic algorithm. These two pictures show the simulation results concluded by the author after implementing the algorithm. 
Reviewed Summary of all Paper

\begin{tabular}{|c|l|l|l|l|l|}
\hline Reference & Network & Evolutionary algorithm & $\begin{array}{l}\text { MANET } \\
\text { Problem }\end{array}$ & \multicolumn{1}{|c|}{ Challenge/s } & Methodology \\
\hline$[21]$ & MANET & $\begin{array}{l}\text { Genetic algorithm; Multi } \\
\text { objective }\end{array}$ & Routing & $\begin{array}{l}\text { To optimizing parameters } \\
\text { through algorithm }\end{array}$ & Simulation \\
\hline$[22]$ & MANET & $\begin{array}{l}\text { Genetic algorithm; Multi } \\
\text { objective }\end{array}$ & Routing & $\begin{array}{l}\text { To improve QOS parame- } \\
\text { ters }\end{array}$ & Simulation \\
\hline$[23]$ & MANET & $\begin{array}{l}\text { Genetic algorithm; Multi } \\
\text { objective }\end{array}$ & Routing & $\begin{array}{l}\text { To improve packet deliv- } \\
\text { ery in the network }\end{array}$ & Simulation \\
\hline$[24]$ & MANET & $\begin{array}{l}\text { Genetic algorithm; Multi } \\
\text { objective }\end{array}$ & Routing & $\begin{array}{l}\text { To secure routing between } \\
\text { nodes through algorithm }\end{array}$ & Simulation \\
\hline$[25]$ & MANET & $\begin{array}{l}\text { Genetic algorithm; Multi } \\
\text { objective }\end{array}$ & Routing & $\begin{array}{l}\text { To optimize energy of } \\
\text { nodes in the network }\end{array}$ & Simulation \\
\hline$[26]$ & MANET & $\begin{array}{l}\text { Genetic algorithm; Multi } \\
\text { objective }\end{array}$ & Routing & $\begin{array}{l}\text { To improve the perfor- } \\
\text { mance of GAODV through } \\
\text { algorithm }\end{array}$ & Simulation \\
\hline$[27]$ & MANET & $\begin{array}{l}\text { Genetic algorithm; Multi } \\
\text { objective }\end{array}$ & Routing & $\begin{array}{l}\text { To improve energy effi- } \\
\text { cient routing }\end{array}$ & Simulation \\
\hline$[28]$ & MANET & Genetic algorithm & $\begin{array}{l}\text { Multicast } \\
\text { Routing }\end{array}$ & $\begin{array}{l}\text { To improve optimization in } \\
\text { jitter, end to end delay and } \\
\text { packet delivery ratio }\end{array}$ & Simulation \\
\hline$[29]$ & MANET & Genetic algorithm & Routing & $\begin{array}{l}\text { To minimize energy con- } \\
\text { sumption in the manet }\end{array}$ & Simulation \\
\hline MANET & Genetic algorithm & Routing & $\begin{array}{l}\text { To find best path for send- } \\
\text { ing data with less cost and } \\
\text { less loss of data }\end{array}$ & Simulation \\
\hline
\end{tabular}

\section{$6 \quad$ Future Direction}

Ad-hoc networks need more experiments in real world application, most of the work presented by different authors have done experiments on different simulators. We can get better network if experiments based on real world of ad-hoc network instead of using only software. MANET Security needs more work in the process of sending information, especially in case of sensitive information like transfer of information between (military). Furthermore, it needs to work more on the Genetic algorithm parameters selections. Routing algorithm should be more powerful in a situation when Network area is big, traffic is more and nodes consume more energy.

Rest of future direction has been taken from Chapter 6 Future trends in MANET by (Arun Biradar).

Congestion control: The Genetic Algorithm concept can be extended to control the congestion in the network.

The concept can be implemented in Qualnet: Qualnet has faster simulation speeds and greater scalability is achievable through smart architecture and optimized memory management of Qualnet.

An adaptive mechanism based on the Genetic Algorithm (GA) is used to control the flow of the data in the transport layer. The Genetic algorithm approach can be used to 
optimize the parameters of a transport protocol to maximize throughput for end-to-end communication over multiple hops.

\section{Conclusion}

We have presented a summary and simulation result of different paper that use GA to overcome the problems of the MANET. The basic concept of GAs is constructed to reproduce the processes in natural system necessary for evolution. The main purpose of this paper to focus on the MANET problems. In this paper, we have studied different problems of MANET that has been solved by the Genetic Algorithm approach. We concluded that the uses of Evolutionary algorithm (GA) can tackle a different kind of problem with possible solutions.

\section{References}

[1] Harmeet Kaur and Maninder Singh "A Survey of Route Optimization Technique in MANET", International Journal of Advanced Research in Electronics and Communication Engineering (IJARECE) Volume 5, Issue 2 February 2016.

[2] D.G.Reina, P.Ruiz, R.Ciobanu, S.L.Toral, B.Dorronsoro, and C.Dobre, "A Survey on the Application of Evolutionary Algorithms for Mobile Multihop Ad Hoc Network Optimization Problems", International Journal of Distributed Sensor Networks Volume 2016, Article ID 2082496. https://doi.org/10.1155/2016/2082496

[3] D. E. Goldberg, "Genetic Algorithms in Search, Optimization, and Machine Learning, Addison Wesley Publishing Company", 1989.

[4] A.A.Mohammad, and Gihan Nagib "Optimal routing in ad hoc networking using Genetic algorithm”, Int.J Advance networking and applications; Vol 03 Issue 05,(2012)

[5] Gihan Nagib and Wahied G. Ali," Network Routing Protocol using Genetic Algorithms", International Journal of Electrical \& Computer Science IJECSIJENS Vol: 10 No: 02, 2010.

[6] Mohapatra, P. and Krishnamurthy, S.V., "Ad Hoc Networks Technologies and Protocols", Springer Verlag, 2005.

[7] Ilyas, M., "The Handbook of Ad Hoc Wireless Networks", CRC Press, Boca Raton, FL, 2003.

[8] https://www.geeksforgeeks.org/ introduction-of-mobile-ad-hoc-network-manet/

[9] D. G. Reina, M. Askalani, S. L. Toral, F. Barrero, E. Asimakopoulou, and N. Bessis, "A survey on multihop ad hoc networks for disaster response scenarios", International Journal of Distributed Sensor Networks, 2015, 16 pages. https://doi.org/10.1155/2015/647037

[10] Internet Engineering Task Force. MANET mobile ad hoc working group. http://www.ietf.org/html.charters/manet-charter.html.

[11] ISHU VARSHNEY, SHAHJAHAN ALI STUDY ON MANET: CONCEPTS, FEATURES AND APPLICATIONS ISSN 2349-9392 (Online); DOI: 10.16962/EAPJMRM/issn. 23499392/2015; Volume 3 Issue 2 (2017)

[12] Aarti. Dr. S. S. and Tyagi, "Study of MANET: Characteristics, Challenges, Application and Security Attacks", International Journal of Advanced Research in Computer Science and Software Engineering, Volume 3, Issue 5, May 2013.

[13] Darwin, C.R., "The Origin of Species "London (John Murray) 1859.

[14] Sivanandam, S. N. and Deepa, S. N. "Introduction to Genetic Algorithms. Springer". (2008). 
[15] Jozef Zurada, "Optimizations problems and Genetic algorithms", University of Louisville USA. Review of Business information system, Volume 14, Third Quarter (2010)

[16] S. Yussof, R.A. Razali and O.H.See, "A parallel genetic algorithm for shortest path Routing Protocol Problem", International conference on future computer and communication, (2009) 268-273. https://doi.org/10.1109/icfcc.2009.36

[17] Mitchell, Melanie, an Introduction to Genetic Algorithms. MIT Press, (1996).

[18] M. Mitchell, An introduction to genetic algorithms. MIT press, 1998.

[19] F. Hillier and G. Lieberman, "Introduction to operations research," McGraw Hill, New York, 2001. [20] M. Ridley, Evolution. 3rd Edition. Blackwell, 2004.

[20] L.M.R.G lobo and S.B chavan, Use of Genetic Algorithm in Network Security, International Journal of computer Application,1-7 (2010).

[21] Redi, D. M. (n.d) "Optimizing Parameters of a Mobile Ad hoc Network Protocol with a Genetic Algorithm," BBN Technologies.

[22] Dr.Ketan, K. S, "Multiobjective Genetic algorithm based adaptive QOS Routing in MANET," IEEE Congress on Evolutionary Computation, 1423-1428 (2007). https:// doi.org/10.1109/cec.2007.4424638

[23] E.Baburaj, V. V, "An Intelligent Multicast Ad-hoc on demand Distance Vector Protocol for MANETs," JOURNAL OF NETWORKS, (2008). https://doi.org/10.4304/jnw.3.6.62-68

[24] D. Suresh. Kumar, "Secure on demand routing protocol for MANET using Genetic Algorithm," International journal of computer applications, 29-34, (2011)

[25] Shenbagaraj R, D. K, "An Optimized and Energy Efficient Multicast Routing Based on Genetic Algorithm for Wireless mobile ad-hoc Networks, "International Journal of Research in IT \& Management, (2012).

[26] Arun Biradar R.C,"Genetic Algorithm Based Unipath and Multipath Intelligent Routing for Mobile Ad-hoc Networks," International Journal of advances in Computer Science and Technology, 276-282 (2014).

[27] Neha Agarwal, Neeraj Manglani, "A new approach for Energy Efficient Routing in Manets Using Multi Objective Genetic Algorithm," International Journal of Science, Engineering and Technology Research (IJSETR) 1780-1784, (2015).

[28] C.Rajan, N.Shanthi, "Genetic Based optimization for multicast routing Algorithm for Manet," Indian Academy of Science, 2341-2352, (2015). https://doi.org/10.1007/s12046-0150437-8

[29] Veena Trivedi, P.N, "Modified AODV Using Genetic Algorithm to minimize Energy consumption in Manet," International Journal of innovative Technology and Exploring Engineering (IJITEE), (2019) 525-530(2019).

[30] Adil M. Salman, I.E "Improving best route using intelligent Ad hoc system," Periodicals of Engineering and Natural Sciences, 824-833(2019). https://doi.org/10.21533/pen.v7i2.513

\section{Authors}

Dr. Jinfa Shi was born in 1963 in china. He received a master's degree from chongqiu Institute of construction engineering in 1989. And received his $\mathrm{PhD}$ degree from Chongqiu University in 1994 respectively. He received his postdoctoral certificate from Beijing Institute of Technology (BIT) in1996. He served as a Lecturer, professor and associate professor in Zhengzhou University of Aeronautics from August 1996 to September 2015.In 2015 he joins North China University of water resources and electric power and serving till now as an associate professor and doctoral supervisor in the 
department of School of management and economy. His research area is Advanced Manufacturing Technology Management, Industrial Engineering and Integrated Management, Information Management System. He published more than 100 papers in these fields. He Won 10 provincial and ministerial achievement awards, Research on the integrated technology of manufacturing information system of manufacturing enterprise in the network environment Henan provincial science and technology progress award. The second prize 2012, as a First Participant. He did research on modern production management systems and its application based on MRP II , Henan provincial science and technology progress award. The second prize 2005, as a First Participant. He presided over 2 NSFC projects as a first participant:

- "Research on enterprise resource set optimization based on the internet of things. "The National Natural Science Foundation Project, 2014(71371172).

- "Research on Modern Manufacturing Fixed Location Management Service Integrated Methodology Based on GIS Principle. "The National Natural Science Foundation Project, 2010(70971120).

Professor Haiyan was born in china. She received her $\mathrm{PhD}$ degree in computer science from Hohai University in 2013. She has been working as a professor at North China University of water resources and electric since 1991. Currently, she is engaged in teaching and scientific research in cloud computing and service computing, optimization selection theory and method, data processing and data mining. As a project leader and main participant, she has successfully completed more than 10 scientific research and engineering project. She has been published more than 20 professional and technical papers in SCI and EI journals.

Article submitted 2020-01-22. Resubmitted 2020-02-26. Final acceptance 2020-02-26. Final version published as submitted by the authors. 